\title{
Design of a dual linear polarization antenna using split ring resonators at $\mathrm{X}$-band
}

\author{
Sadiq Ahmed ${ }^{1,2}$ and Madhukar Chandra ${ }^{1}$ \\ ${ }^{1}$ Department of Microwave Engineering and Electromagnetic Theory, TU Chemnitz, 09126 Chemnitz, Germany \\ ${ }^{2}$ Engineering College, University of Al-Mustansiriyah, Baghdad, Iraq \\ Correspondence to: Sadiq Ahmed (sadiq-kadhim-ahmed.aqbi@s2013.tu-chemnitz.de)
}

Received: 14 December 2016 - Revised: 4 October 2017 - Accepted: 4 October 2017 - Published: 6 November 2017

\begin{abstract}
Dual linear polarization microstrip antenna configurations are very suitable for high-performance satellites, wireless communication and radar applications. This paper presents a new method to improve the co-cross polarization discrimination (XPD) for dual linear polarized microstrip antennas at $10 \mathrm{GHz}$. For this, three various configurations of a dual linear polarization antenna utilizing metamaterial unit cells are shown. In the first layout, the microstrip patch antenna is loaded with two pairs of spiral ring resonators, in the second model, a split ring resonator is placed between two microstrip feed lines, and in the third design, a complementary split ring resonators are etched in the ground plane.

This work has two primary goals: the first is related to the addition of metamaterial unit cells to the antenna structure which permits compensation for an asymmetric current distribution flow on the microstrip antenna and thus yields a symmetrical current distribution on it. This compensation leads to an important enhancement in the XPD in comparison to a conventional dual linear polarized microstrip patch antenna. The simulation reveals an improvement of 7.9, 8.8, and $4 \mathrm{~dB}$ in the $\mathrm{E}$ and $\mathrm{H}$ planes for the three designs, respectively, in the XPD as compared to the conventional dual linear polarized patch antenna. The second objective of this paper is to present the characteristics and performances of the designs of the spiral ring resonator (S-RR), split ring resonator (SRR), and complementary split ring resonator (CSRR) metamaterial unit cells. The simulations are evaluated using the commercial full-wave simulator, Ansoft HighFrequency Structure Simulator (HFSS).
\end{abstract}

\section{Introduction}

Dual linear polarization antennas have seen noteworthy progress in the last few years for several applications such as weather radar and wireless communication systems. The key merit of a dual linear polarization antenna is that provides two independent communication channels, in the ideal case and thus, increasing channel capacity of the communication systems at the same baseband. As such, the development of dual linear polarization antennas has witnessed many studies and designs particularly, in space polarimetric weather radar applications. Therefore, the demand for such systems is growing exponentially (Mishra, 2013; Luo et al., 2013). More importantly, a dual linear polarization antenna suffers from the most significant drawbacks. The first is related to the mutual coupling between two input ports and the second is related to the cross polarization patterns. These drawbacks have provided a fertile ground for the research area. Therefore, many studies suggested and implemented various feeding mechanisms and approach to overcome these two problems and improve the performance of antenna system for their widespread applications (Yang et al., 2013; Liang et al., 2005).

There are many sources that generate cross polarization pattern and mutual coupling between two input ports in dual linear polarization: The higher order modes become one of the main sources of the cross polarization pattern and mutual coupling in dual linear polarized antennas. The second important factor for generating cross-polarization radiation is related to the symmetry of antenna system with respect to the two principal planes which consists of the geometry of the patch antenna and feed network position. Additionally, asymmetry of the current distribution flows on the patch will lead to higher inter-coupling and produces cross polar- 


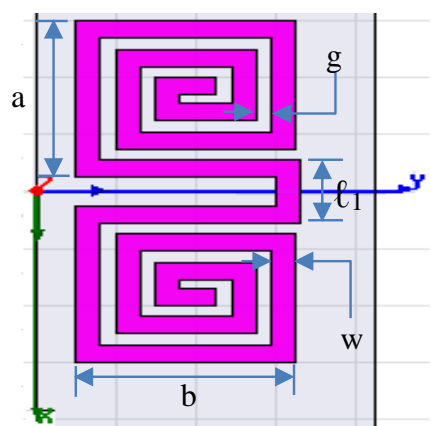

(a)

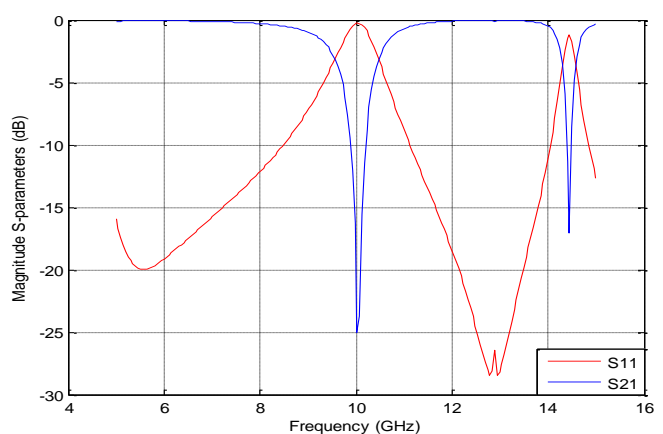

(c)

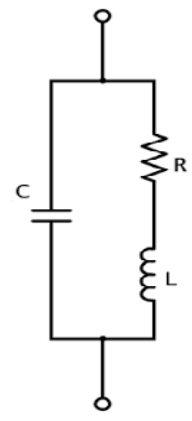

(b)

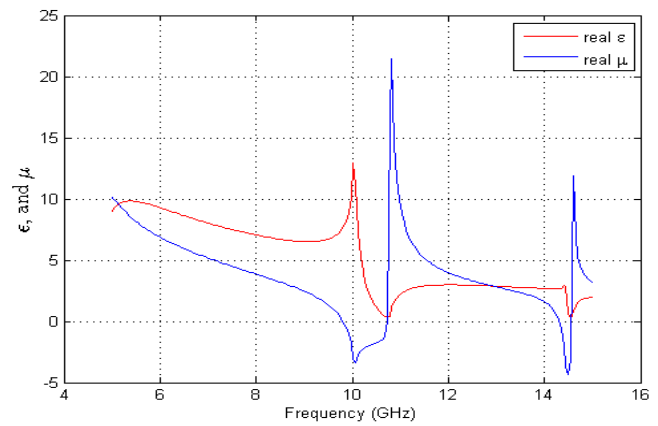

(d)

Figure 1. Spiral ring resonator (a) 2-D geometry of S-RR (b) equivalent circuit (c) $S$-parameters of unit cell (d) permittivity and permeability.

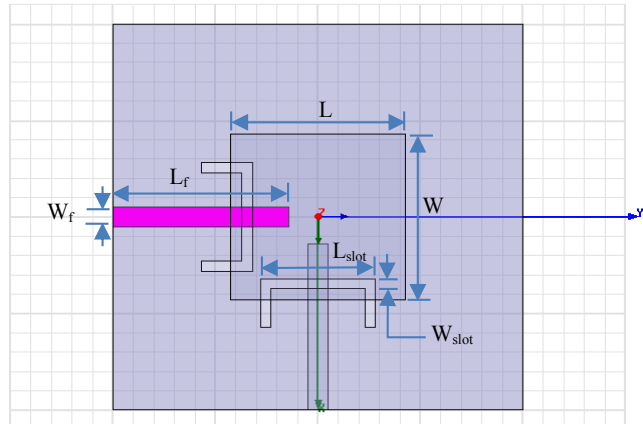

(a)

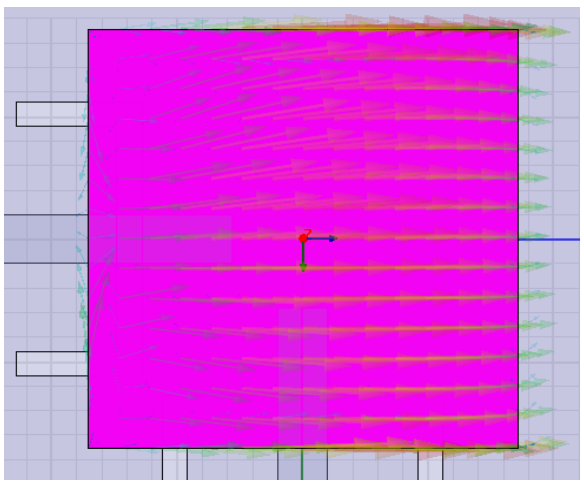

(c)

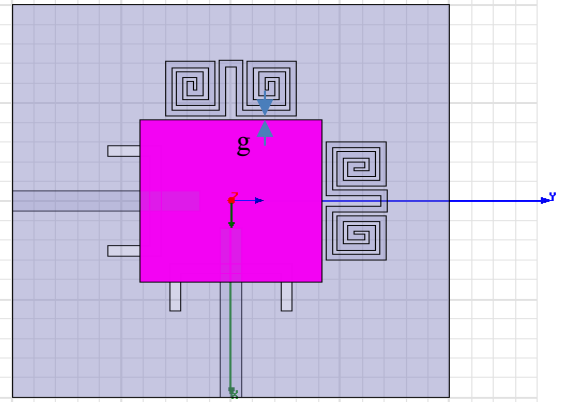

(b)

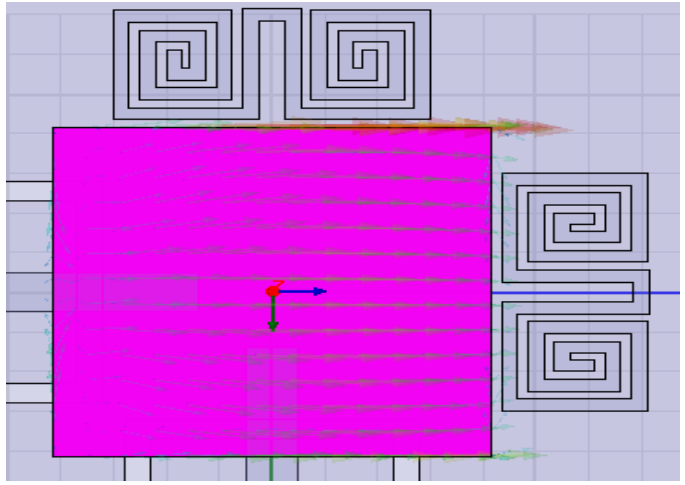

(d)

Figure 2. Dual linear polarization antenna (a) 2-D geometry of conventional dual linear polarization antenna (b) 2-D geometry of proposed antenna (c) current distribution before adding S-RR (d) current distribution due to horizontal port after adding S-RR. 


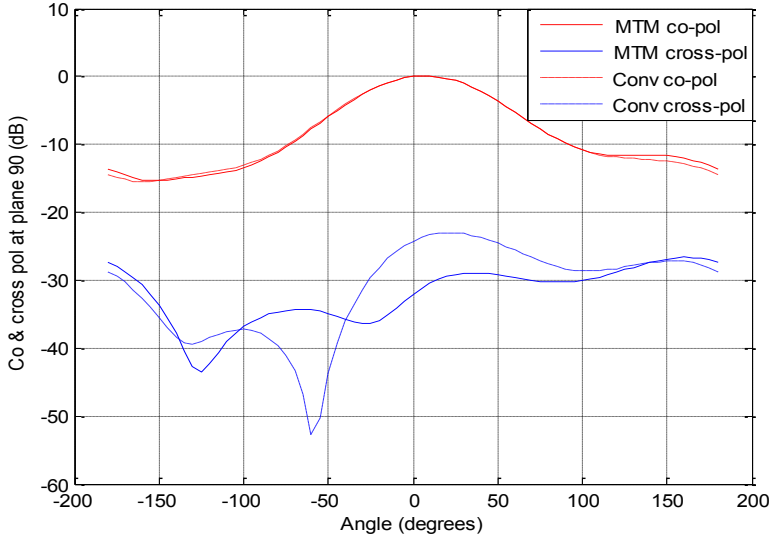

(a)

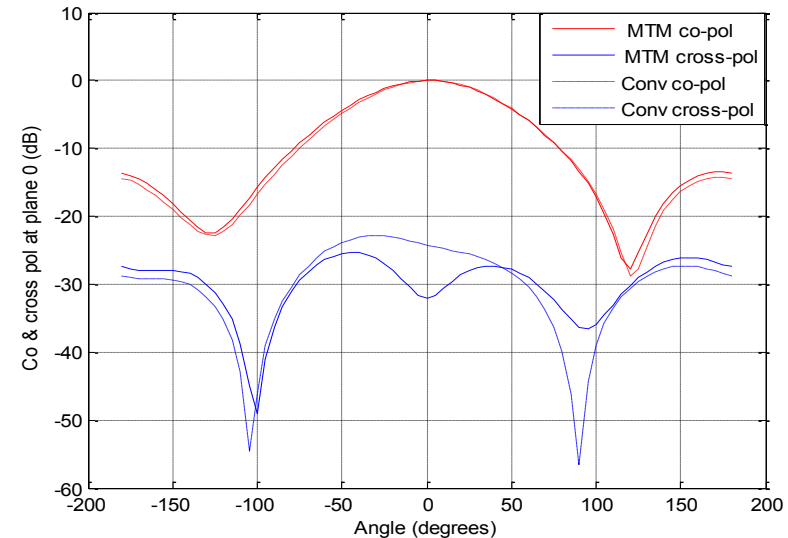

(b)

Figure 3. Co-cross polarization radiation patterns in (a) E (b) H-planes, for a dual polarized antenna with and without metamaterial.

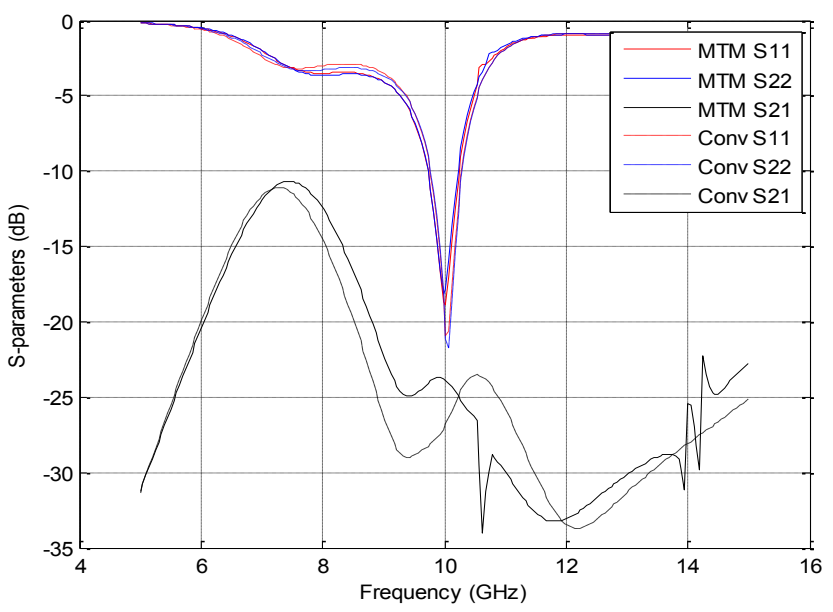

Figure 4. $S$-parameters between two orthogonal input ports with and without S-RR unit cell.

ization pattern. The reason for an asymmetrical current distribution lies in principle characteristic of two perpendicular feeds which place on the nearest two edges while remaining other two edges are without excitation (Liang et al., 2005). To solve this problem, split ring resonators (SRRs) mode of metamaterials are used. Therefore, the SRRs are utilized to compensate the current distribution on the microstrip antenna which leads to improvements in the XPD. Metamaterials (Veselago, 1968) have been used to develop many applications with unique properties (Lin et al., 2004). Metamaterials are utilized in the form of S-RR, SRR, and CSRR structures to represent a single-negative medium and have the properties of a band stop filter (AL-Nuaimi and Whitlow, 2010). This work is organized as follows: the design of a dual linear polarization antenna using a S-RR is presented in Sect. 2. Section 3 is devoted to a dual linear polarization antenna using a SRR design. The design of antenna system
Table 1. Dimensions of a dual linear polarization antenna (all dimensions in $\mathrm{mm}$ ).

\begin{tabular}{rrrrrrrr}
\hline parameters & $L$ & $W$ & $L_{f}$ & $W_{f}$ & $L_{\text {slot }}$ & $W_{\text {slot }}$ & $g$ \\
\hline & 8.3 & 8.3 & 8.6 & 1 & 5.6 & 0.5 & 0.2
\end{tabular}

using CSRRs is presented in Sect. 4. Finally, Sect. 5 contains the conclusions.

\section{Design of a dual linear polarized antenna using a spiral ring resonator}

This part deals with the design of a patch antenna using two S-RRs which are placed close to the radiator patch.

\subsection{Design of a spiral ring resonator as a band stop filter}

The topology of the proposed spiral ring resonator (S-RR) and its equivalent network are illustrated in Fig. 1a and b, respectively. The unit cell consists of two S-RRs joined with a stripline of length $\left(\ell_{1}\right)$ and width $(w)$. The physical dimensions of the S-RR unit cell in our design are: side lengths $a=2.75 \mathrm{~mm}$ and $b=2.75 \mathrm{~mm}$, the gap between metals $g=0.2 \mathrm{~mm}, w=0.3 \mathrm{~mm}$ and $\ell_{1}=1.1 \mathrm{~mm}$. Figure $1 \mathrm{c} \mathrm{de}-$ picts the $S$-parameters of the unit cell; it is observed that the S-RR gives stop band properties $\left(S_{21}=-25 \mathrm{~dB}\right)$ at the center frequency of $10 \mathrm{GHz}$. Figure $1 \mathrm{~d}$ shows the real values of the constitutive parameters $(\varepsilon$ and $\mu)$. It can be recognized that the real values of permeability are purely negative, which means that the metamaterial unit cell works in a single negative medium which leads to a stop band in this region. To validate the $S$-RR performance, it is placed inside the air box and extracted the $S$-parameters and the constitutive parame- 


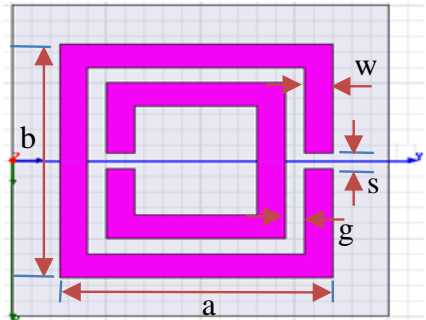

(a)

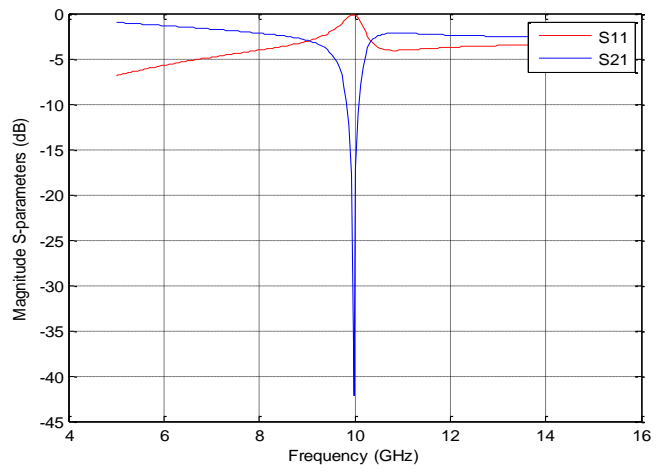

(c)

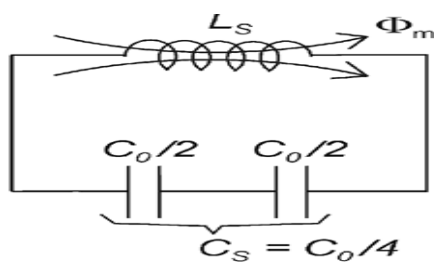

(b)

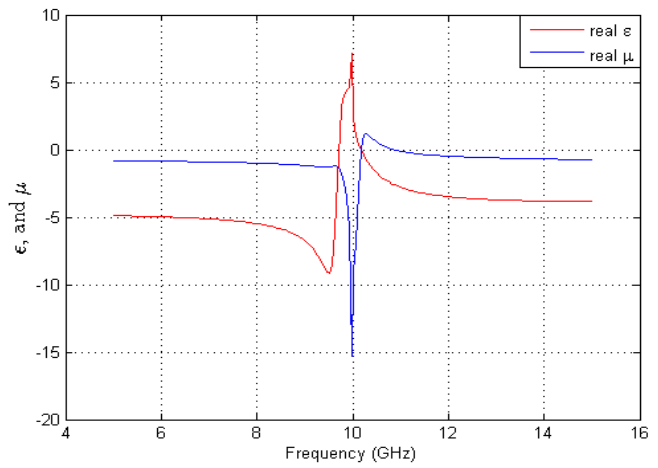

(d)

Figure 5. Split ring resonator (a) 2-D geometry of single split ring resonator (b) equivalent circuit of SRR (c) $S$-parameters of the unit cell $\left(S_{11}\right.$ and $\left.S_{21}\right)$ (d) real values of permittivity, and permeability.

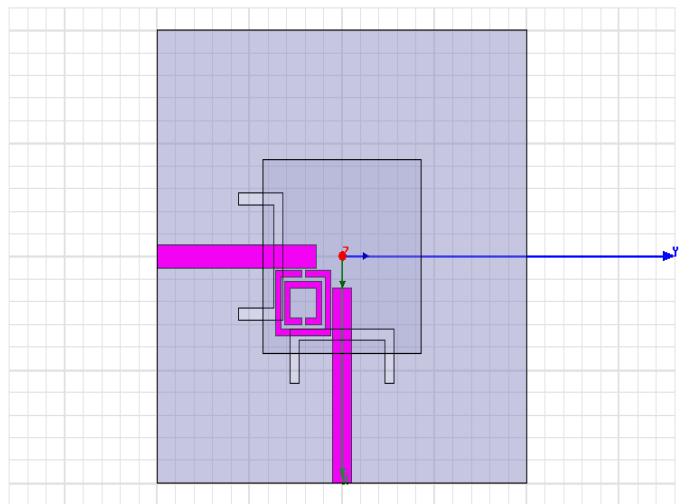

(a)

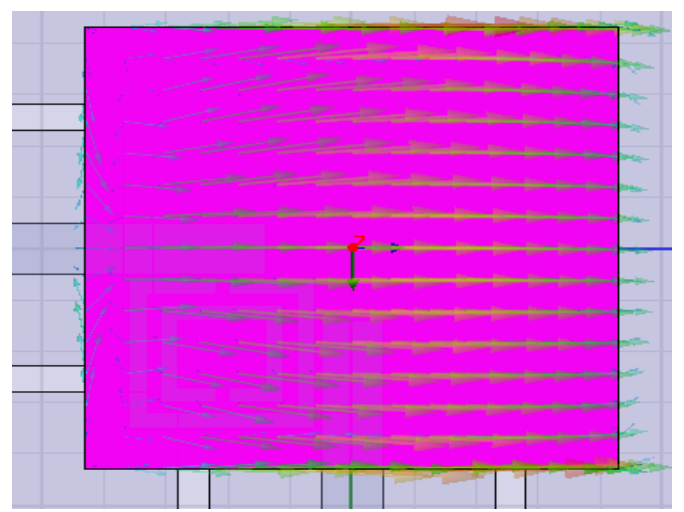

(b)

Figure 6. Geometry of a dual linear polarization antenna (a) 2-D geometry of a dual linear polarization antenna after adding SRR between two microstrip feed lines (b) current distribution due to horizontal port after adding SRR.

ters $(\varepsilon$ and $\mu)$ of unit cell based on the retrieval method as follows (Chen at al., 2004; Sadiq and Chandra, 2016):

$$
\begin{aligned}
& S_{11}=\frac{R_{01}\left(1-e^{j 2 n \beta h}\right)}{1-R_{01}^{2} e^{j 2 n \beta h}} \\
& S_{21}=\frac{\left(1-R_{01}^{2}\right) e^{j 2 n \beta h}}{1-R_{01}^{2} e^{j 2 n \beta h}}
\end{aligned}
$$

$$
\begin{aligned}
z & = \pm \sqrt{\frac{\left(1+S_{11}\right)^{2}-S_{21}^{2}}{\left(1-S_{11}\right)^{2}-S_{21}^{2}}} \\
e^{j n \beta h} & =\frac{S_{21}}{1-S_{11 \frac{z-1}{z+1}}} \\
n & =\frac{1}{\beta h}\left[\left\{\left[\ln \left(e^{j n \beta h}\right)\right]^{\prime \prime}+2 m \pi\right\}\right. \\
& \left.-j\left[\ln \left(e^{j n \beta h}\right)\right]^{\prime}\right]
\end{aligned}
$$


Table 2. Comparison between the traditional dual linear polarization and the proposed antenna using metamaterial with two different examples of $S$-parameters, XPD and BW for two ports.

\begin{tabular}{lrrrrrrr}
\hline ex & $S_{11}(\mathrm{~dB})$ & $S_{22}(\mathrm{~dB})$ & $S_{21}(\mathrm{~dB})$ & $\mathrm{XPD}(\mathrm{dB})$ & $G(\mathrm{~dB})$ & $\mathrm{BW}_{1}(\mathrm{GHz})$ & $\mathrm{BW}_{2}(\mathrm{GHz})$ \\
\hline Conventional Dual pol & -21 & -21.5 & -27 & 24.2 & 7.12 & 0.53 & 0.525 \\
Proposed antenna Ex1 & -19 & -18.5 & -24 & 32.1 & 6.9 & 0.524 & 0.501 \\
Proposed antenna Ex2 & -21.2 & -21 & -26.3 & 33 & 7.1 & 0.535 & 0.5323 \\
Proposed antenna Ex3 & -22.4 & -31.7 & -25 & 28.2 & 7.8 & 0.533 & 0.622 \\
\hline
\end{tabular}

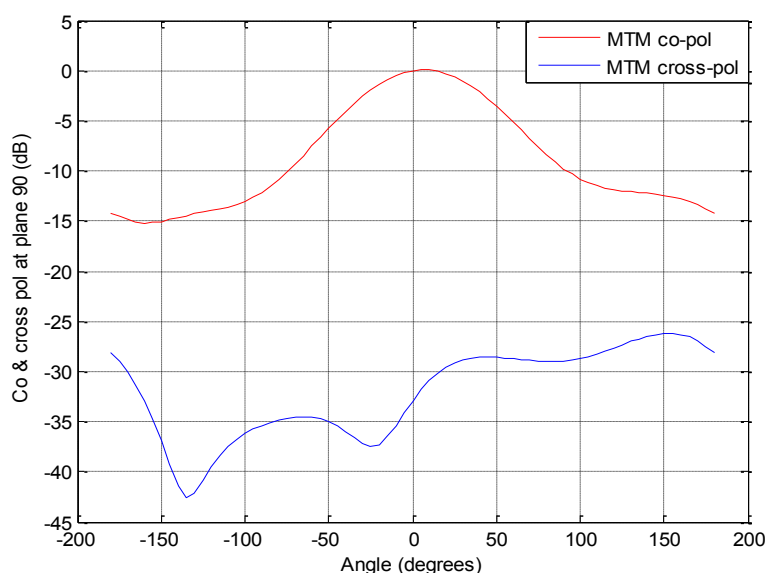

(a)

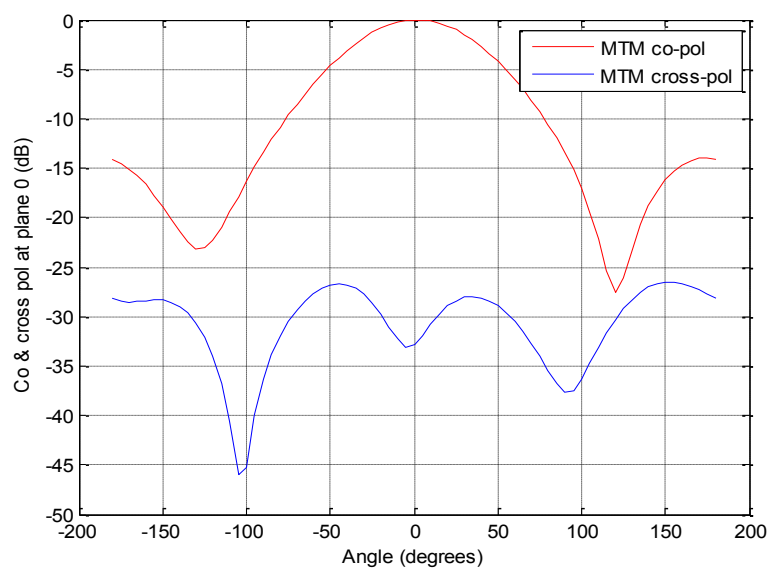

(b)

Figure 7. Co-cross polarization radiation patterns in (a) E (b) H-planes, for a dual linear polarization with metamaterial.

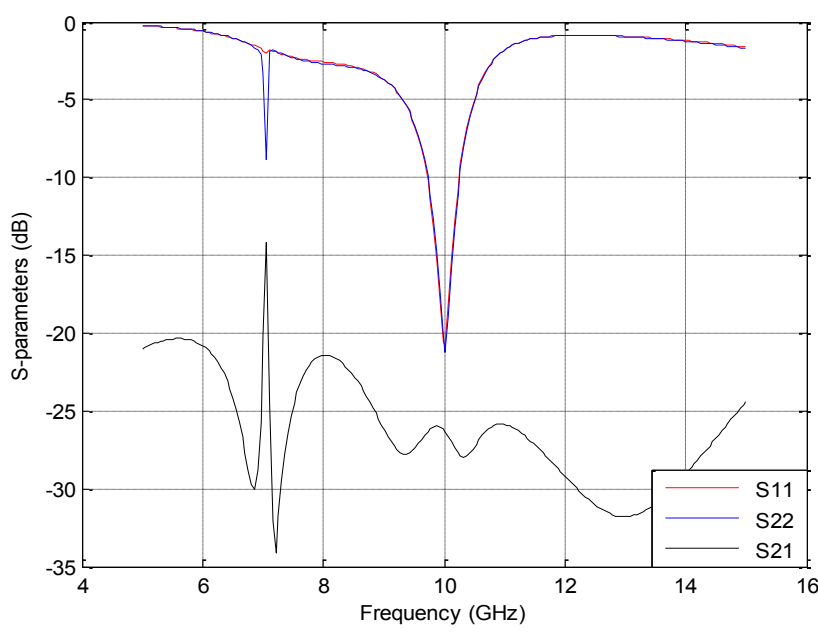

Figure 8. $S$-parameters between two orthogonal input ports with SRR unit cell.

where (.) represents the real component and (.) $)^{\prime \prime}$ the imaginary part of the complex number; $S_{11}$ and $S_{21}$ are the reflection and transmission coefficients, respectively; $n$ is the refractive index; $R_{01}$ is $(z-1) /(z+1) ; z$ is the impedance; $m$ is the branch due to the periodicity of the sinusoidal function; $\beta$ is the wave number constant; $h$ is the height of sub- strate material. Permeability and permittivity can be written by the following equations (Numan and Sharawi, 2008):

$$
\begin{aligned}
\mu_{r} & =n z \\
\varepsilon_{r} & =n / z
\end{aligned}
$$

\subsection{Design of a dual linear polarization antenna}

After the design of S-RR unit cell, we have inserted two unit cells into dual linear polarized antenna system. The 2-D top view structure of the proposed a dual linear polarization patch antenna at $10 \mathrm{GHz}$ is shown in Fig. $2 \mathrm{a}$ and $\mathrm{b}$ before and after the incorporation of the two spiral ring resonators. The microstrip antenna and unit cells are printed on the first substrate layer made-up of Roger RT/duroid 5880 substrates with a relative permittivity of 2.2 , a height of $1.575 \mathrm{~mm}$, and loss tangent $\tan \delta=0.0009$. Two orthogonal aperture microstrip feed lines represent the feeding systems, which are placed beneath the second substrate layer that utilize Rogers RO4350 substrate with a thickness of $0.508 \mathrm{~mm}$, the relative permittivity of 3.48, and loss tangent $\tan \delta=0.004$. The ground lies between two substrate materials, which involve two apertures. Determination of the current distribu- 


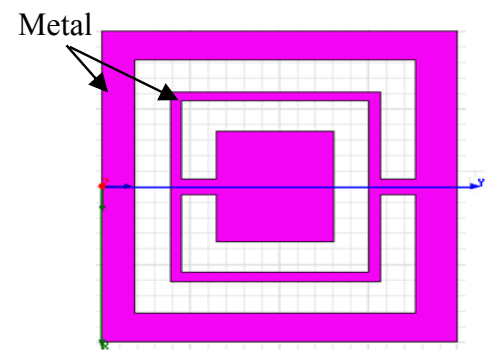

(a)

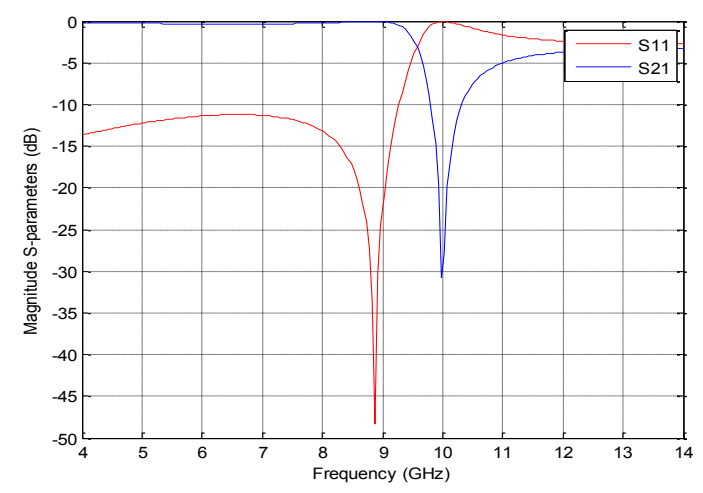

(c)

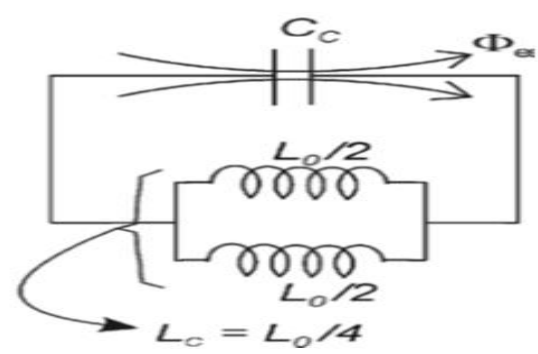

(b)

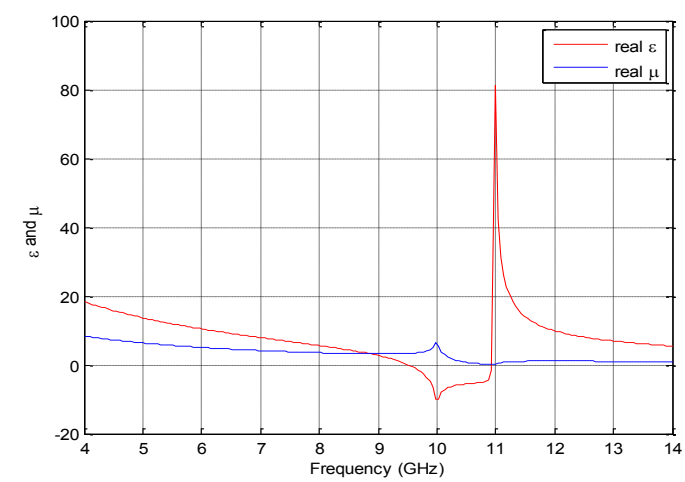

(d)

Figure 9. Complementary split ring resonator (a) 2-D geometry of a CSRR (b) equivalent circuit of a CSRR (c) $S$-parameters of the unit cell $\left(S_{11}\right.$ and $\left.S_{21}\right)(\mathbf{d})$ real values of permittivity, and permeability.

tion along the proposed patch antenna provides a good indication for improving the XPD. It is observed that the current distribution becomes more symmetric after adding the two S-RRs as depicted in Fig. 2c and d, which show the current distribution before and after the addition of unit cells due to horizontal port. With such a setup, the XPD can be enhanced.

Figure $3 \mathrm{a}$ and $\mathrm{b}$ displays the normalized co-cross polarization radiation patterns in principle planes for conventional and proposed dual linear polarized antennas. It is observed that a significant improvement in the XPD in comparison to the original dual linear polarized patch antenna (without S-RRs). The simulation reveals an increase of $7.9 \mathrm{~dB}$ in the XPD as compared to the conventional antenna. The reflection and isolation coefficients between two orthogonal input ports with and without S-RR inclusions are shown in Fig. 4. It is observed that the isolation becomes worse by $3 \mathrm{~dB}$ and the return loss for two input ports are $S_{11}=-19 \mathrm{~dB}$ and $S_{22}=-18.5 \mathrm{~dB}$ at the resonance frequency. The dimensions of a dual linear polarized antenna system are listed in Table 1.

\section{Design of a dual linear antenna using a split ring resonator with a frequency of $10 \mathrm{GHz}$}

This section presents the design of a dual linear polarization microstrip antenna with two orthogonal aperture-coupling microstrip feed lines. In this design, the SRRs are placed between two microstrip feed lines.

\subsection{Design of the SRR as a Band Stop Filter}

Figure 5a and $\mathrm{b}$ shows the 2-D structure of the SRR which consist of two concentric square rings and its equivalent circuit, respectively. The metamaterial structure has been implemented on a RO4350C high-frequency laminate $\left(\varepsilon_{r}=3.48\right.$, the thickness of the substrate is $0.508 \mathrm{~mm}$ and a metal thickness of the copper cladding of $35 \mu \mathrm{m}$ ). The geometry of SRR is optimized in order to be used with a feeding system for a dual linear polarized patch antenna.The parameters of this filter are: side lengths $a=3 \mathrm{~mm}, b=2.9$, gap between metals $g=0.2$, slot width $s=0.2 \mathrm{~mm}$, and width of stripline $w=0.3 \mathrm{~mm}$.

The radiation of microstrip feed lines will interfere with the radiation of the patch and this interference will generate higher order modes and thus leads to the generation of cross polarization patterns. To solve this problem, metamaterial inclusions are placed between the two microstrip feed lines and thus absorb some of the microstrip line radiation, therefore, suppress the higher order modes and leading to more symmetry in current distribution and finally will improve the XPD. Based on this idea, SRRs have been designed as a band stop microstrip filter which absorbs the waves radiated from 


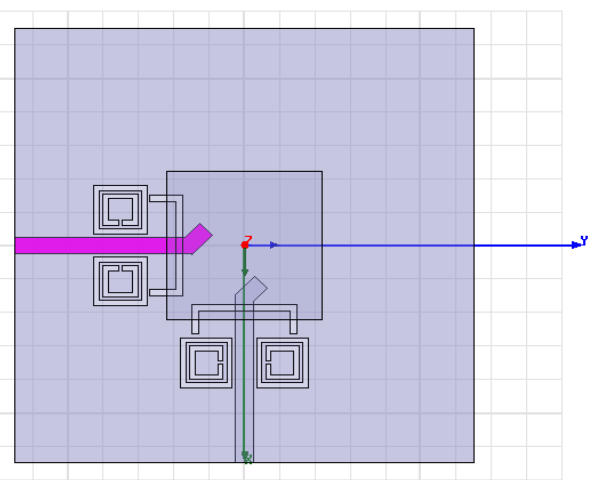

(a)

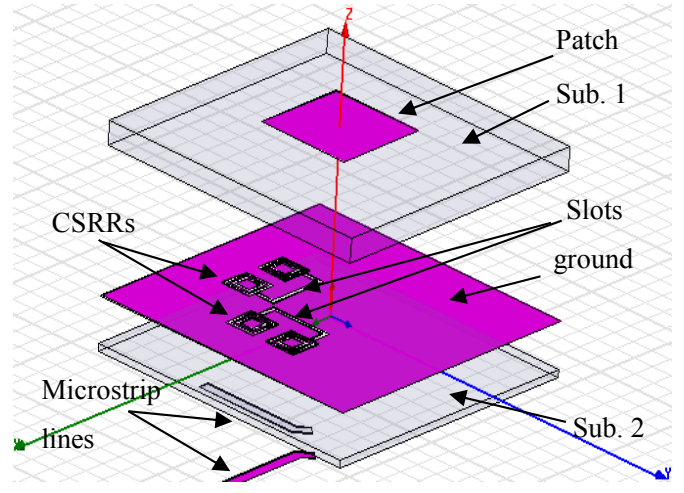

(b)

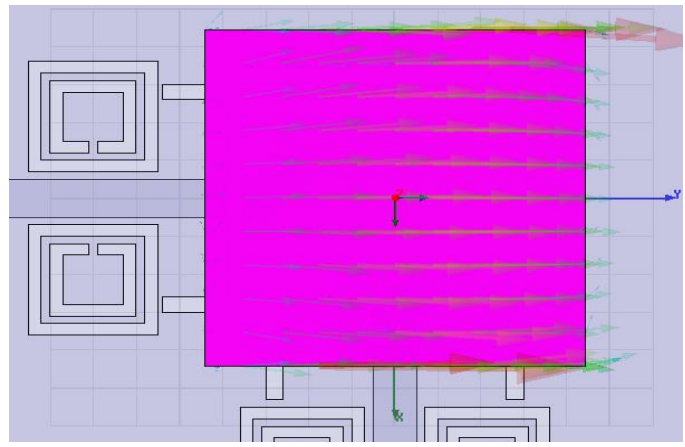

(c)

Figure 10. Geometry of a dual linear polarization antenna (a) 2-D geometry of a dual linear polarization antenna after adding a pair of CSRR (b) 3-D structure of the antenna system (c) Current distribution flow on the patch antenna after adding CSRR.

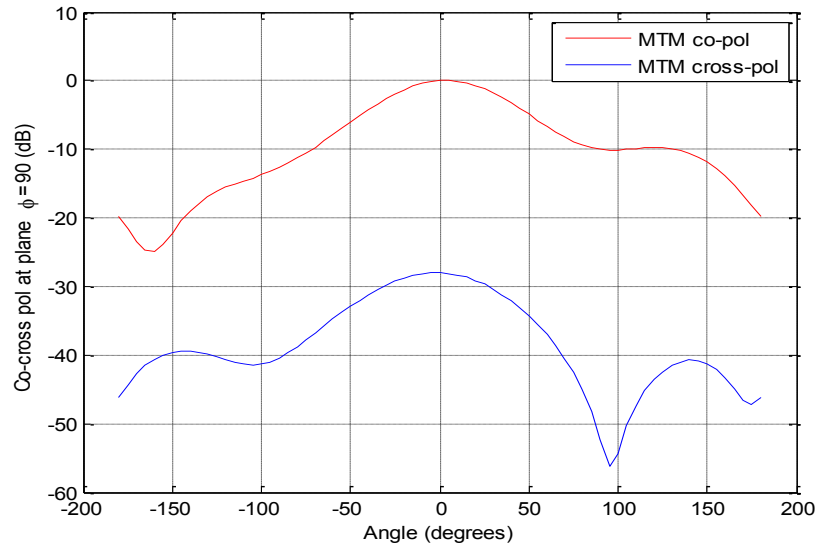

(a)

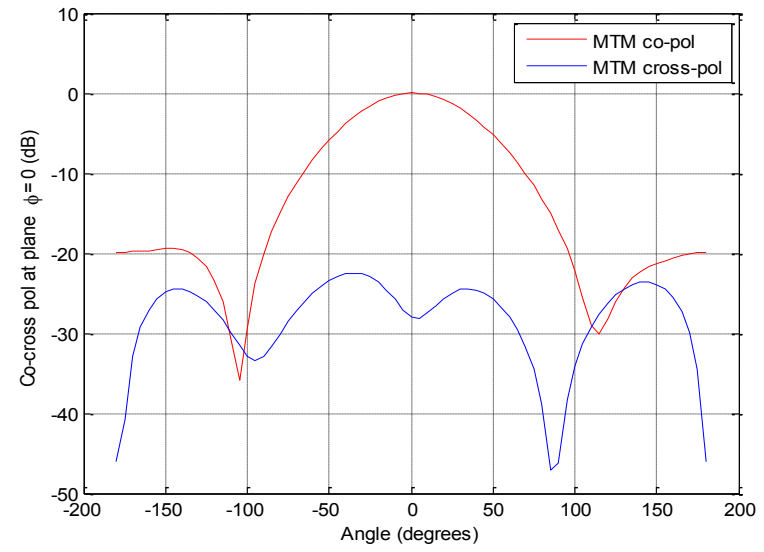

(b)

Figure 11. Linear co-cross polarization radiation patterns in (a) E- (b) H-planes, for a dual linear polarization with metamaterial.

two microstrip feed lines. The microstrip feed has a width of $1 \mathrm{~mm}$, which is corresponding to a characteristic impedance of $50 \Omega$. Figure 5 c displays the $S$-parameters of the unit cell. It can be seen that SRR provides to stop band properties $\left(S_{21}=-41 \mathrm{~dB}\right)$ at the center frequency of $10 \mathrm{GHz}$. The real values of the constitutive parameters $(\varepsilon$ and $\mu)$ are plotted in Fig. 5d, it is noteworthy that the real values of permeability are purely negative which leads to a stop band in this region. 


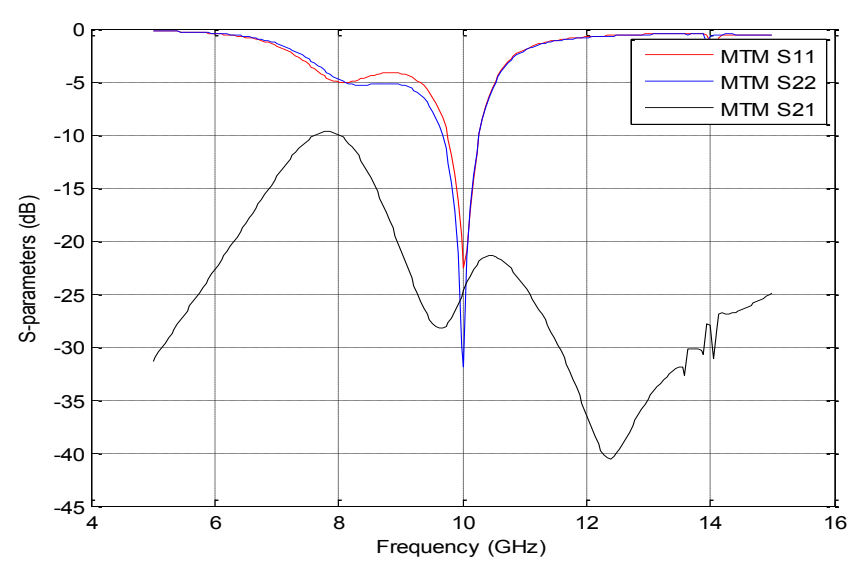

Figure 12. $S$-parameters between two orthogonal input ports.

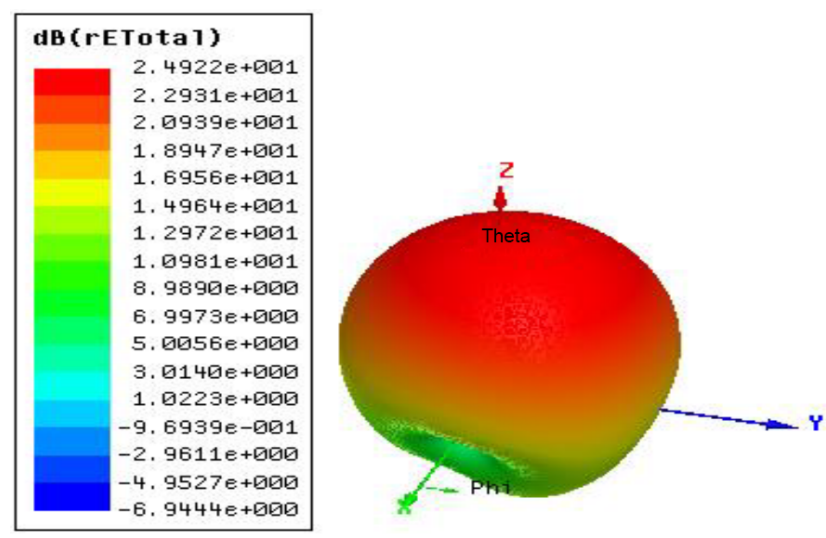

Figure 13. 3-D radiation pattern of antenna system.

\subsection{Design of a dual linear polarization antenna using split ring resonators between two microstrip lines}

In this part, a SRR is placed between two microstrip feed lines as shown in Fig. 6a. The space between SRRs and the microstrip feed line is $0.1 \mathrm{~mm}$. More symmetry in the current distribution is observed after adding of unit cells as shown in Fig. 6b. Linear, co-cross polarization patterns in the $\mathrm{E}$ and $\mathrm{H}$ planes are depicted in Fig. 7a and b, which shows a significant improvement of the XPD in comparison to the conventional dual linear polarized antenna. The simulation reveals an improvement of $8.8 \mathrm{~dB}$ in the XPD as compared to the conventional antenna. Figure 8 shows the reflection and mutual coupling coefficients between two orthogonal input ports. It is noticed that the mutual coupling becomes a little worse by $0.7 \mathrm{~dB}\left(S_{21}=-26.3 \mathrm{~dB}\right)$ and the reflection coefficients for the two input ports are $S_{11}=-21.2$ and $S_{22}=-21 \mathrm{~dB}$ at the center frequency as demonstrated in Table 2 .

\section{Design of a dual linear antenna using a complementary split ring resonator with a frequency of $10 \mathrm{GHz}$}

This part presents the design of a microstrip antenna with two orthogonal aperture-coupling microstrip feed lines. In this design, the complementary split ring resonators (CSRRs) are used.

\subsection{Design of a complementary split ring resonator as a Band Stop Filter}

The complementary split ring resonator (CSRR) is performed by etching SRR in the ground plane as illustrated in Fig. 9a. It has approximately the same shape of the SRR structure. The CSRRs display inverse properties of those of the SRRs. For example, The SRR works as a magnetic dipole while the CSRR behaves as an electric dipole. Thus CSRR produces negative permittivity $(\operatorname{Re}(\varepsilon)<0)$ instead of negative permeability $(\operatorname{Re}(\mu)<0)$ near the resonance frequency. Figure $9 \mathrm{~b}$ shows the equivalent circuit of CSRR (Al-Nuaimi and Whitlow, 2010). All dimensions of the CSRRs have been chosen identical to their SRR counterparts so that the operating frequency of the band stop filter is also around $10 \mathrm{GHz}$. The distance between the microstrip feed line and the CSRRs is determined by the height of the substrate material. Figure 9c displays the $S$-parameters of a CSRR, it is observed that CSRR offers to stop band properties $\left(S_{21}=-30 \mathrm{~dB}\right)$ at the transition frequency of $10 \mathrm{GHz}$. The real values of the constitutive parameters $(\varepsilon$ and $\mu)$ are shown in Fig. $9 \mathrm{~d}$ and it may be noticed that the real values of permittivity are purely negative which leads to a band-rejection filter in this region.

\subsection{Design of a dual linear polarization antenna using two pairs of complementary split ring resonators (CSRR)}

Figure 10a and $\mathrm{b}$ shows the 2-D and 3-D geometry of a dual linear polarization antenna using two pairs of the CSRR unit cells, respectively. It is noticed that more symmetry in the current distribution flows on radiator patch after adding of the two pairs of unit cells as shown in Fig. 10c. Figure 11a and $b$ shows the linear co-cross polarization radiation patterns in the $\mathrm{E}$ and $\mathrm{H}$ planes at the broadside. The simulation reveals that the XPD of $28.2 \mathrm{~dB}$, which means that enhancement of $4 \mathrm{~dB}$ in the XPD as compared to the conventional antenna. Figure 12 shows the reflection and mutual coupling coefficients between two orthogonal input ports with two pairs of CSRRs. It is observed that the mutual coupling becomes worse by $2 \mathrm{~dB}\left(S_{21}=-25 \mathrm{~dB}\right)$ and the input reflection coefficients for the two input ports are $S_{11}=-22.4$ and $S_{22}=-31.7 \mathrm{~dB}$ at the resonance frequency as demonstrated in Table 2. Figure 13 shows the 3-D radiation pattern of the antenna system. 


\section{Conclusions}

In this paper, a novel approach (using metamaterials) is used to enhance the XPD for a dual linear polarization patch antenna at the frequency of $10 \mathrm{GHz}$. This improvement is obtained by placing two $\mathrm{S}$-RRs close to the microstrip patch antenna, placing a SRR between two microstrip feed lines, and etching two pairs of CSRRs in the ground plane. An improvement in the XPD by $8.8 \mathrm{~dB}$ as compared to the conventional dual linear polarization antenna is noticed. The antenna system has several advantages involving a simple structure, and metamaterial inclusions occupied the very small area, which makes the proposed metamaterial more useful for the design of dual linear polarization antennas. The proposed antenna is very compact as compared to a conventional dual linear polarization antenna and has the desired improved characteristics.

Data availability. The paper depends on the design of antenna using software and Matlab program.

Competing interests. The authors declare that they have no conflict of interest.

Special issue statement. This article is part of the special issue "Kleinheubacher Berichte 2016". It is a result of the Kleinheubacher Tagung 2016, Miltenberg, Germany, 26-28 September 2016.

Edited by: Gerd Wanielik

Reviewed by: three anonymous referees

\section{References}

Al-Nuaimi, M. K. T. and Whitlow, W. G.: Compact microstrip bandstop filter using SRR and CSSR: Design, simulation, and results, Antennas Propag. (EuCAP), Proc. Fourth Eur. Conf., 2-6, 2010.

Chen, X., Grzegorcezyk, T. M., Wu, B., Pacheco, J., and Kong, J. A.: Robust Method to Retrieve the Constitutive Effective Parameters of Metamaterials, Physics Review E, 70, 1-7, 2004.

Liang, X. L., Zhong, S. S., and Wang, W.: Design of A Dual Polarization Microstrip Patch Antenna with excellent Polarization Purity, Microw. Opt. Techn. Let., 44, 329-331, 2005.

Lin, I. H., DeVincentis M., Caloz, C., and Itoh T.: Arbitrary dualband components using composite right/left-handed transmission lines, IEEE T. Microw. Theory, 52, 1142-1149, 2004.

Luo, K., Ding, W., Hu, Y., and Cao, W.: Design of Dual-Feed DualPolarized Microstrip Antenna with High Isolation and Low Cross Polarization, Progress In Electromagnetics Research Letters, 36, 31-40, 2013.

Mishra, P. K., Jahagirdar, D. R., and Kumar, G.: An Array of Broadband Dual Polarized Electromagnetically Coupled Microstrip Antennas, Prog. Electromagn. Res., 44, 211-223, 2013.

Numan, A. B. and Sharawi, M. S.: Extraction of Material Parameters for Metamaterials Using a Full-Wave Simulator, IEEE Antenn. Propag. M., 55, 202-211, 2013.

Sadiq, A. and Chandra, M.: Cross Polarization Discrimination Enhancement of a Dual Linear Polarization Antenna Using Metamaterials, UKSim-AMSS 18th International Conference on Computer Modelling and Simulation, 366-371, 2016.

Veselago, V.: The electrodynamics of substances with simultaneously negative values of $\varepsilon$ and $\mu$, Soviet Physics Uspekhi, 10, 509-514, 1968.

Yang, X., Gong, B., Tan G., and Lu, Z.: Reconfigurable patch antennas with Four polarization States Agility Using Dual Feed Ports, Prog. Electromagn. Res., 54, 285-301, 2013. 